\title{
Una actividad experimental fundamental sobre efecto Hall
}

\author{
A fundamental experimental activity on Hall effect
}

\author{
Daniel Baccino*1,2@, Sergio Leopoldo Falcón ${ }^{3}$, Guzmán Pablo Trinidad ${ }^{3}$ \\ ${ }^{1}$ Instituto de Profesores Artigas, Departamento Académico Nacional de Física, Montevideo, Uruguay \\ ${ }^{2}$ Liceo N 10 , Dr. Carlos Vaz Ferreira, Laboratorio de Física, Montevideo, Uruguay \\ ${ }^{3}$ Liceo Solymar 1, Ciudad de la Costa, Canelones, Uruguay
}

Recibida en 9 de December, 2017. Revisado en 6 de Abril, 2018. Aceptado en 18 de Mayo, 2018.

\begin{abstract}
Es poco frecuente encontrar dispositivos de bajo costo que muestren el efecto Hall en laboratorios de física de enseñanza media. Presentamos un montaje y una actividad experimental asociada en la que se mide dicho efecto en bismuto. La importancia tecnológica y científica de fenómenos de interacción entre campos y corrientes fundamenta la inclusión de su tratamiento en los cursos básicos de física.

Palabras clave: Efecto Hall, Bismuto, Física experimental, Enseñanza de la física.
\end{abstract}

It is rare to find low-cost devices that show the Hall effect in middle school physics laboratories. We present a montage and an associated experimental activity in which this effect is measured in bismuth. The technological and scientific importance of phenomena of interaction between fields and currents bases the inclusion of its treatment in the basic courses of physics.

Keywords: Hall Effect, Bismuth, Experimental Physics, Physics Teaching.

\section{Introducción}

El físico estadounidense Edwin Herbert Hall publica, en 1879, el artículo On a New Action of the Magnet on Electric Currents [1]. Rescatamos de ese artículo la descripción de uno de los montajes con los que trabajó (la traducción es libre):

Con el fin de realizar experimentos cuantitativos aproximados, se preparó una nueva plancha consistente en un hoja de oro de aproximadamente $2 \mathrm{~cm}$ de ancho y $9 \mathrm{~cm}$ de largo montada en una placa de vidrio. Se aseguró un buen contacto presionando firmemente hacia abajo en cada extremo de la tira de la hoja de oro un grueso pedazo de latón pulido en el lado inferior. A estos pedazos de latón se soldaron los alambres de una sola celda de Bunsen. La porción de la hoja de oro no cubierta por las piezas de latón era de unos $5,5 \mathrm{~cm}$ de longitud y tenía una resistencia de alrededor de 2 ohmios. Los polos de un galvanómetro Thomson de alta resistencia se conectaron con puntos opuestos entre sí en los bordes de la hoja de oro y a medio camino entre los pedazos de latón. La placa de vidrio que llevaba la hoja de oro fue fijada, como la primera, por un cemento blando al extremo plano de un polo del imán, y el otro polo del

*Endereço de correspondência: dbaccisi@gmail.com imán fue llevado a aproximadamente $6 \mathrm{~mm}$ de la hoja de oro.

Uno de los motivos por los cuales incluimos esta cita algo extensa del trabajo de Hall es que no hay grandes diferencias cualitativas con el montaje que hemos utilizado en nuestro trabajo.

El lector puede encontrar fácilmente en la web material donde se plantean propósitos cualitativos o cuantitativos para mostrar y/o analizar el efecto. A modo de ejemplo anotamos la referencia [2].

\section{Elementos de la teoría sobre el efecto Hall}

Un enfoque en profundidad sobre este efecto puede leerse en Kittel [3] donde se plantea un desarrollo basado en el modelo "gas de electrones", para una muestra que tiene un sólo tipo de portadores de carga pasibles de definir una corriente eléctrica sobre una muestra que se imagina prismática, como se ve esquemáticamente en la figura 1.

Nuestro planteo elemental se sustenta en el de Halliday - Resnick [4], que comienza:

En 1879, Edwin H. Hall llevó a cabo un experimento que permitió la medición directa del signo y la densidad del número (número por unidad de volumen) de los portadores de carga en un conductor. El efecto Hall desempeña un papel crítico en nuestra comprensión 


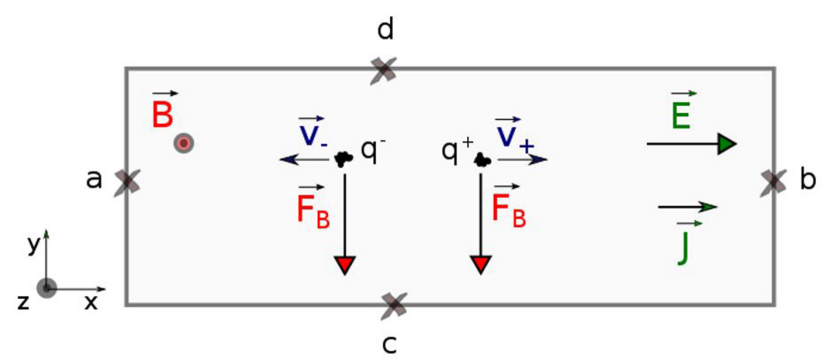

Figura 1: Geometría estándar para estudiar el Efecto Hall.

de la conducción eléctrica en los metales y semiconductores.

En un párrafo posterior, refiriéndose a la posibilidad de identificar el tipo de portadores de la muestra, afirman:

Esto puede parecer una conclusión obvia en el caso de los metales; sin embargo, deberá tenerse en cuenta que el trabajo de Hall se hizo casi 20 años antes del descubrimiento del electrón por Thomson, y la naturaleza de la conducción eléctrica en los metales no era en absoluto obvia en aquel tiempo.

\subsection{Identificación de los portadores de carga eléctrica}

En la figura 1 se muestra esquemáticamente una sección normal al eje $z$ de una muestra prismática de material. La misma se coloca en una zona de campo magnético $B_{z}$ (para fijar ideas tomemos $B_{z}>0$ ). Un campo eléctrico $E_{x}$ aplicado a lo largo de la varilla (dirección longitudinal, con $E_{x}>0$ ) produce una densidad de corriente $J_{x}$. Esta corriente tiene asociado el movimiento de portadores de carga eléctrica que, en principio, podrían tener carga positiva $\left(\mathrm{q}^{+}\right)$, negativa $\left(\mathrm{q}^{-}\right)$, o ambos.

La presencia del campo magnético origina que los portadores se acumulen en la cara inferior del esquema, mientras que en la cara opuesta se genera un exceso de iones de signo opuesto al de los portadores desviados. Establecido un régimen, el efecto del campo Hall se compensa con la fuerza debida al campo magnético, mientras los portadores adquieren una velocidad de arrastre $v$.

Observe que, en nuestro modelo y con $B_{z}>0$, los portadores (independientemente del tipo que sean) experimentan una fuerza magnética orientada hacia la cara a la que pertenece el punto c. Este hecho notable permite identificar el tipo de portadores (mayoritarios en el caso de que estén presente ambos) mediante el signo de la diferencia de potencial transversal $\mathrm{V}_{\mathrm{cd}}$.

\subsection{Voltaje de Hall en términos de magnitudes medibles}

A partir de la situación de régimen se puede plantear la relación:

$$
q \cdot \vec{E}+q \cdot \vec{v} \times \vec{B}=0
$$

En nuestro modelo la ecuación anterior termina siendo:

$$
E_{y}=v_{x} \cdot B_{z}
$$

En el Anexo I se desarrolla una expresión para escribir a la velocidad de arrastre $\mathrm{v}$ en términos de la densidad de corriente $J_{x}$, y una ecuación en la que aparecen varias magnitudes medibles macroscópicamente:

$$
V_{H}=-\frac{1}{n e} \frac{1}{\Delta z} \cdot I \cdot B_{z}
$$

En la ecuación 1, $V_{H}$ es el voltaje transversal (voltaje Hall) observado entre los puntos c y d, $\Delta z$ el espesor de la lámina, $\mathrm{n}$ la densidad de portadores, e la carga elemental y $B_{z}$ la componente del campo magnético generado por un imán no dibujado y que asumimos uniforme y única.

Observamos que, si se puede medir independientemente $V_{H}, \Delta z, I$ y $B_{z}$, es posible determinar la densidad $\mathrm{n}$ de portadores en la muestra.

Nuestro modelo elemental predice una proporcionalidad directa entre $V_{H}$ y $B_{z}$ si se mantiene fija la intensidad de corriente por la muestra y una relación del mismo tipo entre $V_{H}$ e $I$, asegurando un $B_{z}$ fijo.

\subsection{El efecto en el bismuto}

A partir de la ecuación 1 vemos que, para valores dados de $\Delta z, I$ y $B_{z}$, el fenómeno es tanto más visible cuanto mayor sea el factor $(-1 /$ ne). Esto es relevante porque, si tiene un valor relativamente grande, permitirá medir el voltaje $V_{H}$ con instrumentos y técnicas elementales. Es usual llamarlo coeficiente Hall,

$$
R_{H} \equiv-\frac{1}{n e}
$$

El bismuto, un semimetal diamagnético, presenta portadores de carga tanto positivos como negativos con diferente concentración. Tiene asociado un factor $R_{H}$ relativamente grande $\left(-5.4 \times 10^{-7} \mathrm{~m}^{3} / \mathrm{C}\right)$. Kittel sostiene que tal condición no es explicable mediante el modelo "gas de electrones"; se requiere de la teoría de bandas de energía para hacerlo.

En nuestro modelo asumimos la existencia de un solo tipo de portadores. En tal caso su concentración es relativamente baja, de forma que el efecto se torna relevante. Este es uno de los motivos por los que elegimos el material de la muestra.

En el caso general, al someter bismuto a un campo magnético, se producen fenómenos complejos cuya explicación excede un tratamiento elemental. Vea, por ejemplo, la referencia [5]. Sin embargo el diseño que presentamos arroja resultados consistentes con el modelo propuesto.

\section{Un dispositivo experimental para estudiar el efecto}

\subsection{La placa de bismuto}

Es importante decir que no se consigue bismuto fácilmente en Uruguay pero puede adquirirse a precio razonable 
en el exterior y recibirse por correo. Se trata de un metal tan frágil y quebradizo que no puede cortarse ni martillarse al intentar laminarlo sin que se desgrane en trozos pequeños. Para obtener una lámina delgada de espesor pequeño y uniforme se puede someter la muestra de bismuto al fuego directo de mechero Bunsen (por ejemplo) y dejar caer las gotas desde una altura de $1.0 \mathrm{~m}$ (aproximadamente) a la superficie de un vidrio previamente limpiado. Como resultado se obtienen "gotas estalladas" cuyo aspecto recuerda a las de estaño que conocemos perfectamente en nuestros laboratorios al trabajar con el soldador. Ambos aspectos se ven en la figura 2.

Se recomienda obtener varias de estas "gotas" y medir sus espesores: se elegirán aquellas que presenten espesor regular y de valor mínimo (por lo general del orden de $0.10 \mathrm{~mm})$.

\subsection{Montaje del dispositivo}

Se recortan por lo menos dos de las gotas seleccionadas en forma de rectángulo (en adelante muestra o plaquita de bismuto) y se les sueldan ${ }^{1}$ (en a y b de la figura 1) dos alambres muy largos (previamente identificados con colores diferentes). Estos conectores se utilizarán para conectar la plaquita a la fuente de alimentación y a un amperímetro, lo que permite ajustar y medir la intensidad de corriente eléctrica que circulará en la dirección "longitudinal" de la misma (la plaquita se dispone de tal forma que dicha dirección será la horizontal). Se somete una de las plaquitas a voltaje continuo creciente para averiguar el valor de intensidad máxima que soporta antes de fundirse. Una vez averiguado dicho valor se sueldan los conectores de alimentación a la otra plaquita

\footnotetext{
1 Los terminales de cobre se soldaron a la muestra de bismuto con un soldador y estaño. El objetivo de esta técnica fue optimizar el contacto entre la muestra y los conectores.
}

seleccionada (que será la muestra a medir) de similares características para trabajar con valores por debajo de aquel máximo. Otros dos alambres (previamente identificados con colores diferentes) se sueldan a la muestra (en c y d) para conectarlos a un voltímetro que resuelva hasta un máximo de $0.1 \mathrm{mV}$. Esta medida de voltaje "transversal", junto al voltaje de offset (ver más adelante en la sección 4) nos permitirá calcular el Voltaje Hall $\left(V_{H}\right)$. Vea una imagen del dispositivo en la figura 3 (izquierda).

La muestra de bismuto y sus cuatro conectores se fijan y montan sobre una placa plana de material no conductor como, por ejemplo, cualquier placa universal para montaje de componentes electrónicos. Los conectores que alimentan la muestra deben alejarse perpendicularmente a la arista y en la dirección de su plano. Una vez fuera de la placa aislante se tornean entre sí, con el objetivo de minimizar el campo magnético que producen; esto es importante por el método utilizado para calcular el campo magnético del imán. Vea la figura 3 (derecha).

Es importante destacar que tanto la muestra como las conexiones eléctricas como para el montaje y sujeción de la placa, fueron no ferromagnéticos.

Para la imposición y medida del campo magnético a la que está sometida la muestra disponemos de un par de imanes rectangulares de neodimio montados sobre una $U$ de hierro y una balanza que resuelve hasta un máximo de $0.01 \mathrm{~g}$, respectivamente. La determinación del módulo de la inducción magnética $B_{z}$ se hace, asumiendo homogeneidad, mediante la técnica desarrollada en "Balanza de Cotton" [6].

En el Anexo II se muestra un mapeo previo del campo $B_{z}$ en la zona en la que se ubica la muestra; y en el Anexo III se muestra una corrección que podría tener que realizarse en las medidas de masa inusuales para una balanza.
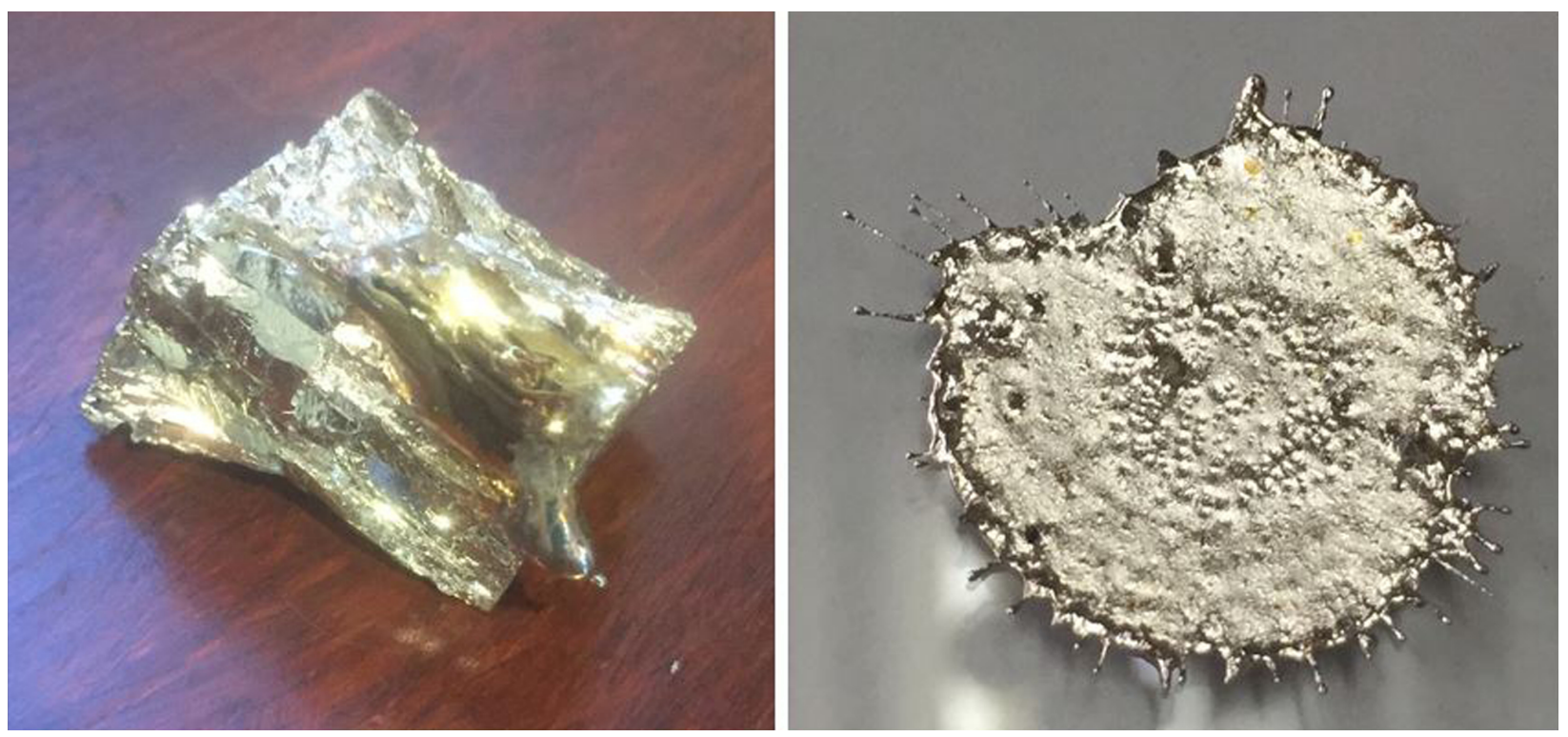

Figura 2: Trozo de bismuto (izquierda); gota "estallada" de bismuto (derecha) 

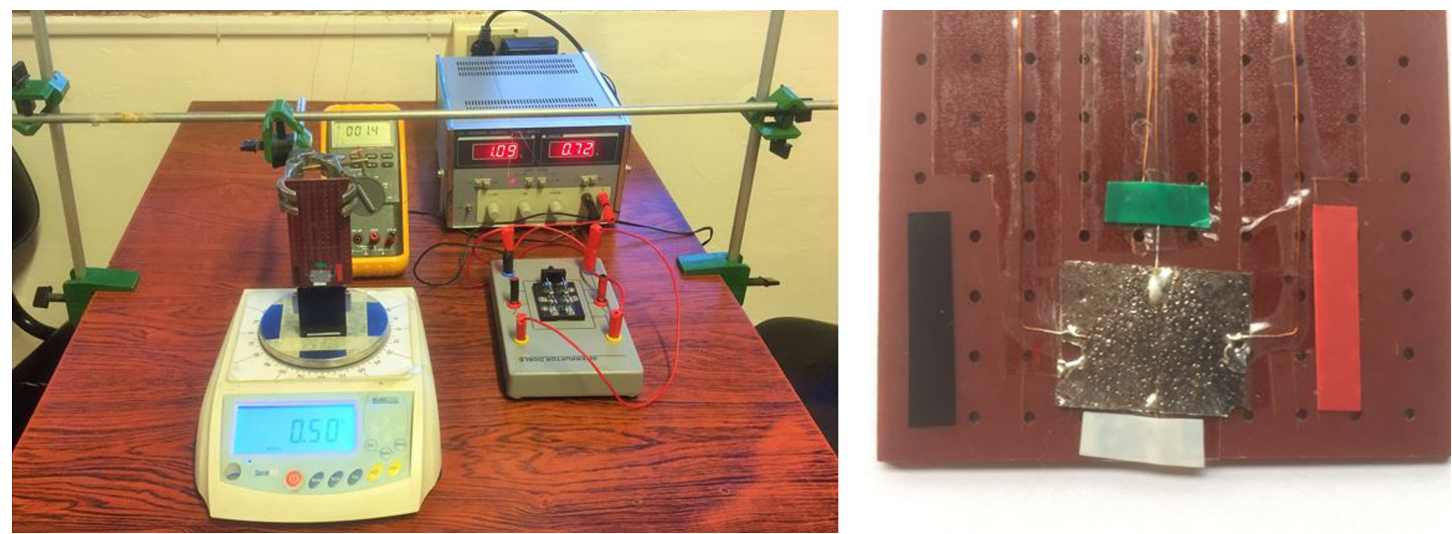

Figura 3: Dispositivo (izquierda). Muestra montada sobre una placa no conductora (derecha)

\section{Nuestros propósitos y primeros resultados}

Nos planteamos en este trabajo determinar el signo de los portadores mayoritarios de carga en la muestra, y desde un punto de vista cuantitativo, las dos relaciones funcionales que se esbozan en el modelo elemental en la sección Elementos de la teoría.

En primer lugar nuestra atención se concentró en la determinación del signo de la diferencia de potencial entre los puntos c y d en la figura 1. Este signo es consistente con el tipo de portadores mayoritarios (negativos) que se espera tener en el bismuto.

Un aspecto que hay que considerar al realizar la medida del voltaje transversal entre c y d es que usualmente encontramos una diferencia de potencial entre estos puntos en ausencia de campo magnético. Este corrimiento se debe, seguramente, a una desalineación de los conectores que puede explicarse fundamentalmente en función de su magnetorresistencia (vea, por ejemplo, la página 498 de la referencia [3] $)^{2}$ Lo llamamos voltaje offset y lo consideramos un error sistemático a corregir. En el Anexo V se muestra la necesidad de esta corrección.

\subsection{Con campo magnético fijo}

Un resultado obtenido, al dejar fijo el campo magnético en el valor $B_{z}=(0.28 \pm 0.02) \mathrm{T}$ en la zona en la que se dispone la muestra de $\mathrm{Bi}$, se presenta en el gráfico de la figura 4.

Un ajuste lineal al conjunto completo de datos (se relevó la corriente en ambos sentidos) origina el resultado siguiente:

$$
\begin{gathered}
\text { Pendiente }=(-1.252 \pm 0.009) \mathrm{mV} / \mathrm{A} \\
\text { Ordenada en el origen }=(-0.02 \pm 0.01) \mathrm{mV}
\end{gathered}
$$

El coeficiente de correlación de Pearson de valor muy cercano a -1 por una parte, y la ordenada en el origen

\footnotetext{
2 Una definición de magnetorresistencia aplicada a la geometría de nuestro problema y sus consecuencias, pueden consultarse en el Problema 4 (pág. 299) de la 2da edición de Kittel.
}

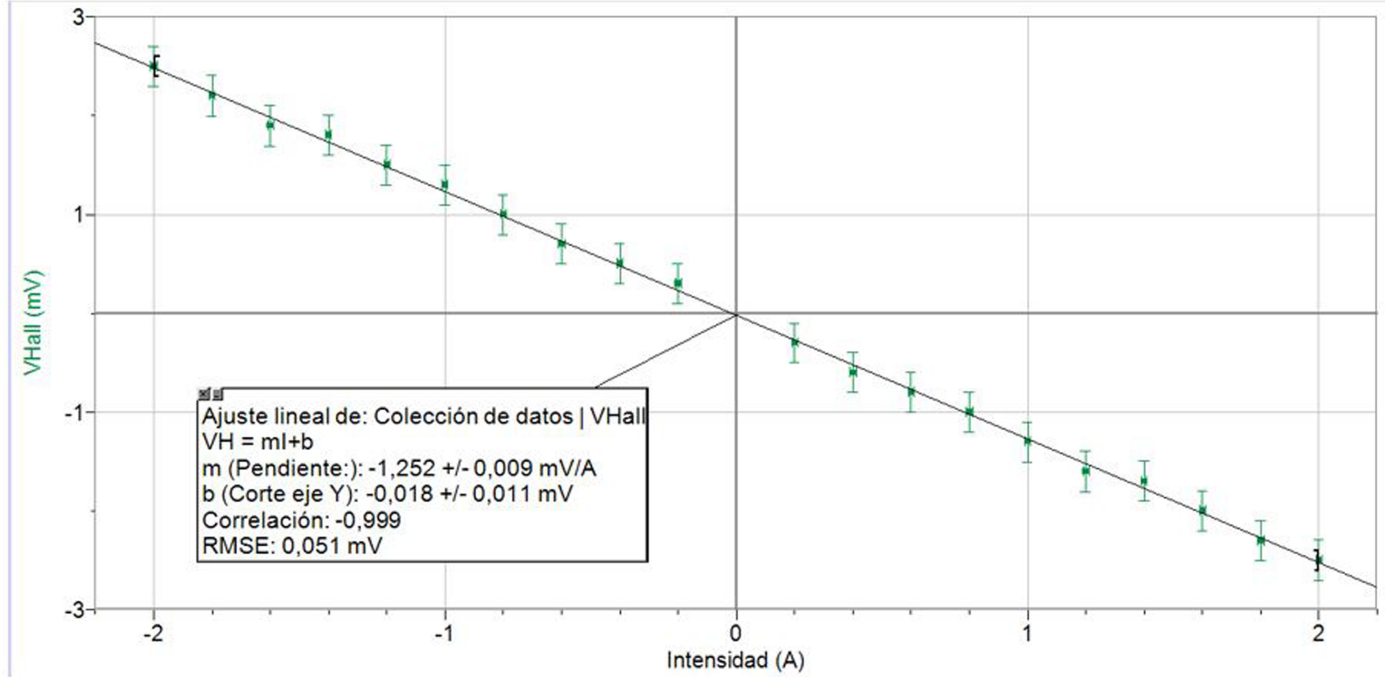

Figura 4: Voltaje Hall al variar la intensidad de corriente, con campo B fijo. 


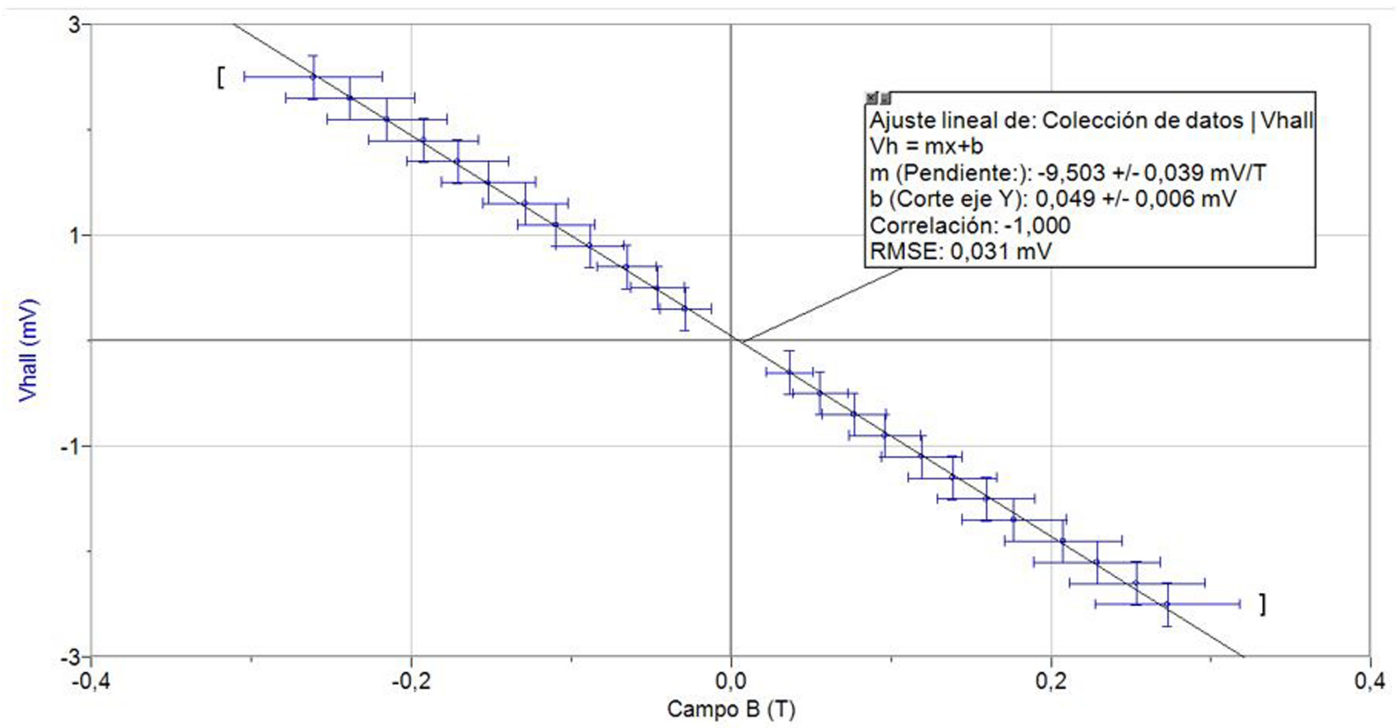

Figura 5: Voltaje Hall al variar el campo B, con intensidad de corriente fija.

y el valor RMSE de un orden de magnitud menor a la incertidumbre en la medida de $V_{H}$ por el otro, nos permiten concluir que existe una proporcionalidad directa entre las magnitudes graficadas $V_{H}$ eI.

\subsection{Resultados con corriente fija}

La figura 5 muestra nuestros resultados para un caso en el cual, dejando fija la intensidad de corriente, se sometió a la muestra a un campo $B_{z}$ variable en el rango que nos permitió el dispositivo.

Los resultados cuantitativos más destacados del ajuste son:

$$
\text { Pendiente }=(-9.50 \pm 0.04) \mathrm{mV} / \mathrm{T}
$$

Ordenada en el origen $=(0.049 \pm 0.006) \mathrm{mV}$

Los resultados permiten concluir que existe una proporcionalidad directa entre las magnitudes graficadas

$$
V_{H} y B_{z} \text {. }
$$

\section{Comentarios finales}

Una forma de presentar sintéticamente los resultados cuantitativos es determinar el coeficiente $R_{H}$ a partir de cada uno de los coeficientes principales de los ajustes presentados en las figuras 4 y 5 utilizando la ecuación 2 .

Los valores de $R_{\mathbf{H}}$ obtenidos de esta forma son: $-4.9 \mathrm{x}$ $10^{-7} \mathrm{~m}^{3} / \mathrm{C}$, para el caso de campo incambiado y $-5.2 \mathrm{x}$ $10^{-7} \mathrm{~m}^{3} / \mathrm{C}$ para el caso de intensidad de corriente fija.

\section{Agradecimientos}

Agradecemos al Prof. Eduardo Núñez y al Dr. Ricardo Marotti, sus valiosos aportes, y a dos instituciones que nos han permitido desarrollar este trabajo: Liceo Solymar 1, y Liceo $N^{\circ} 10$ Dr. Carlos Vaz Ferreira.

\section{Material suplementario}

El siguiente material suplementario está disponible en línea:

Anexo 1- Expresión de $\mathrm{V}_{\mathrm{H}}$ a partir de un modelo elemental

Anexo II - Mapeo del campo en la zona de trabajo

Anexo III - Corrección del error sistemático en la calibración de la balanza digital al medir "masas negativas" Anexo IV - Ficha técnica del dispositivo experimental Anexo V-Corrección del offset

\section{Referencias}

[1] E. H. Hall, American Journal of Mathematics 2, 287 (1879)

[2] University of Colorado, The Hall Effect, accesible en $<$ https://www.colorado.edu/physics/phys2150/ phys $2150 \_f a 13 / 12 \% 20-\% 20$ The $\% 20 \mathrm{Hall} \% 20$ Effect $\%$ 20 (Exp.\%2012\%20Edited).pdf >.

[3] C. Kittel, Introducción a la Física del Estado Sólido (Reverté, Barcelona, 1995), p. 177, $3^{\text {a }}$ ed.

[4] D. Halliday, R. Resnick, Física (CECSA, México, 1994), v. 2, p. 169.

[5] S. Field, The Hall Effect, accesible en $<$ https://pdfs.semanticscholar.org/07c7/ 15bc7267f6dd73e2d6b479277c2e302d039d.pdf >

[6] G. Trinidad, D. Baccino, Balanza de Cotton, accesible en $<$ http://www.uruguayeduca.edu.uy/sites/default/ files/2017-05/BALANZA\%20DE\%20COTTON_vf.pdf > 